Article

\title{
The Frequency Selective Effect of Radar Backscattering from Multiscale Sea Surface
}

\author{
Dengfeng Xie ${ }^{1,2}\left(\mathbb{D}\right.$, Kun-Shan Chen ${ }^{1,3, *} \mathbb{C}$ and Jiangyuan Zeng ${ }^{1}$ \\ 1 State Key Laboratory of Remote Sensing Science, Institute of Remote Sensing and Digital Earth, Chinese \\ Academy of Sciences, Beijing 100101, China; xiedf@radi.ac.cn (D.X.); zengjy@radi.ac.cn (J.Z.) \\ 2 University of Chinese Academy of Sciences, Beijing 100049, China \\ 3 School of Land Resources and Urban and Rural Planning, Hebei GEO University, \\ Shijiazhuang 050000, China \\ * Correspondence: chenks@radi.ac.cn; Tel.: +86-139-1063-4643
}

Received: 10 December 2018; Accepted: 12 January 2019; Published: 16 January 2019

check for updates

\begin{abstract}
The sea surface essentially contains multiscale roughness with capillary waves of many sizes riding on large-scale waves that are also of many sizes. It is instructive to exploit the effect of radar frequency and observation geometry on the effective roughness scales responsible for radar backscattering so that the scattering mechanism and the scattering source can be better understood and quantitated. Based on common sea spectra and a theoretical scattering model, an attempt is made to attain the above objective. Model predictions, with selective roughness scales, are compared with wide validation data, including L-band radar observations, and predictions from C-band and $\mathrm{Ku}$-band empirical models: geophysical model function (CMOD7) and NASA scatterometer (NSCAT-4) for C- and Ku-bands at different incident angles. Numerical results indicate that effective roughness scales for radar backscattering vary with radar frequency and incidence angle and are related to a portion of sea spectral components; the low limit of which is linearly proportional to the Bragg wavenumber determined by frequency and incidence angle, and the scale factor of the linear relationship is about 0.05 . In addition, the root mean square (RMS) height and the correlation length of the effective roughness (i.e., scattering source) derived from the effective roughness decrease gradually as incident angle increases. In particular, the correlation length also linearly depends on the effective wavelength with a coefficient of 3.2. Moreover, these two coefficients are both independent of wind speed, radar frequency, and incident angle. These findings also reveal the essential properties of the spectral components contributing to radar backscattering and its variation with radar frequency and incident angle.
\end{abstract}

Keywords: radar scattering; effective roughness scale; Bragg wavenumber; sea spectrum

\section{Introduction}

The sea surface possesses an anisotropic, non-Gaussian height, and a multiscale rough surface due to the effect of local wind and the waves propagating from other parts of the sea [1-3]. The modulation of different scales complicates the radar scattering from the sea surface that deserves a deeper look. A good understanding of the scattering mechanism is essential and useful for retrieving the geophysical parameters of the sea surface [4-6].

Over the past decades, attempts have been made to understand the mechanism and behavior behind the scattering, analytical modeling, numerical simulation, and experimental measurement [7]. In the study of radar backscattering from rough surfaces, most work focuses on the single-scale rough surfaces. Numerous numerical simulations and controlled laboratory experiments have been carried out to understand the nature of surface backscattering [8,9]. It is found that surface backscattering 
from a single-scale rough surface is dependent on the surface root mean square (RMS) height and its correlation function (or all orders of its roughness spectrum). In contrast, the backscattering from multiscale rough surface depends on many scales of roughness and its scattering mechanism has been studied in references [9-12]. These studies indicate that the scales of roughness responsible for radar backscattering are dependent on incident angles at a given exploring frequency.

For multiscale sea surface, the surface roughness (i.e., correlation function and RMS height) is usually characterized by a sea surface height spectrum model determined by oceanographers. In the Two Scale Model (TSM), the sea spectrum is divided into large- and small-scale roughness in order to apply the Kirchhoff Approximation (KA) and Small Perturbation Model (SPM), respectively, and thus only the two roughness scales are assumed to be responsible for backscattering. Various choices exist for the dividing wavenumber $k_{d}$, ranging from $k / 1.5$ to $k / 40: k_{d}=k / 1.5$ [13]; $k_{d}=k / 3 \sim k_{d}=k / 6$ [14]; $k_{d}=k / 5 \sim k_{d}=k / 10$ [15]; $k_{d}=k / 40$ [16], where $k$ is the wavenumber. These heuristic dividing wavenumbers suggest that with only two roughness scales, perhaps it is hard to explain the roughness effect on radar backscattering, with a more complete picture from multiscale sea surface, which is usually characterized by the effective roughness, in light of radar backscattering [9]. In order to simplify the backscattering calculation, Fung et al. $[9,12]$ approximated the sea surface correlation function of the sea surface to an exponential-like function. The corresponding roughness parameters (e.g., correlation length and RMS height) in the correlation function (surface height spectrum) were determined by fitting the backscattering simulations predicted by the Integral Equation Model (IEM) with the radar measurements. Nevertheless, this approximation perhaps is unlikely to find out the contributing wave components responsible for radar backscattering. To the best of our knowledge, limited studies have quantitatively investigated whether size of scale wave or sea surface spectral components does contribute to radar backscattering and how it varies with the combinations of incident angles and exploring frequencies. Therefore, the main purpose of this paper is to quantitatively estimate the radar frequency selective effect of effective roughness responsible for radar backscattering through the comparison of the simulated backscattering based on AIEM model, the advanced version of IEM, and sea spectrum models with the radar observations or empirical models derived from extensive experimental data. This detailed and quantitative analysis of the selective effect is theoretically significant to deepen and expand the understanding of backscattering mechanism from multiscale sea surface and guides us towards more effective, and perhaps more accurate, retrieval of ocean parameters (e.g., sea wind field).

In this paper, the roughness scale responsible for radar backscattering was generated from a partial of sea spectral components and was investigated quantitatively based on the AIEM and the existing sea spectrum models. Firstly, the correlation functions and roughness parameters of various roughness constructed by partial sea spectrum components were calculated theoretically. Then, the roughness scale contributing to radar backscattering was quantitatively investigated and assessed by matching the corresponding measured data at the specific observation geometry and exploring frequency (L-, C- and Ku-bands). In Section 2, the sea spectrum and AIEM model along with the validation data used in this paper are briefly introduced. Section 3 illustrates the sea surface roughness parameters (i.e., RMS height and autocorrelation function) derived from a part of spectral components in sea spectrum model. Then, in Section 4 the backscattering from sea surface with various roughness was simulated by AIEM and the effective roughness for radar backscattering was determined by the comparison of simulated backscattering coefficients with the corresponding observations or empirical models. Section 5 further analyzed the effect of sea spectrum models on backscattering simulations. Finally, the concluding remarks are given in Section 6.

\section{Models and Validation Data}

In this section, the sea spectra and AIEM model, used for simulating backscattering, along with the validation data for examining the effective roughness for radar backscattering from the multiscale sea surface are shown briefly. 


\subsection{Sea Spectrum Model}

The unifying two-dimension directional sea spectrum mainly contains two parts: one is the omnidirectional spectrum $S(K)$, representing the isotropic part; the other is the angular spreading function (ASF) [17], allocating the energy of each spectral component to all azimuth directions. At present, the common omnidirectional sea spectrum models contain D-V spectrum [18], Apel spectrum [19], and Elfouhaily spectrum [17], etc. They were widely used for topography simulation of sea surface to simulate scattering cross section from sea surface $[20,21]$. The two-dimension directional sea spectrum is defined in polar coordinates as:

$$
S(K, \psi)=\frac{1}{K} S(K) \Phi(K, \psi)
$$

where

$$
\Phi(K, \psi)=[1+\Delta(K) \cos (2 \psi)] / 2 \pi
$$

In Equations (1) and (2), $K$ represents the spectral components and ranges from 0 to positive infinity in theory; $\psi$ is the azimuth angle and $\psi=0^{\circ}$ is in upwind direction. The RMS height $(\sigma)$ and normalized autocorrelation function of sea surface elevation $\rho(r, \varphi)$ are directly related to the sea spectrum components by the following definition:

$$
\begin{gathered}
\sigma^{2}=\int_{0}^{\infty} S(K) d K \\
\rho(r, \varphi)=\frac{1}{\sigma^{2}} \int_{0}^{\infty} \int_{0}^{2 \pi} S(K, \psi) \exp (j K r \cos (\varphi-\psi)) K d K d \psi
\end{gathered}
$$

Similar to the two-dimension sea spectrum, the correlation function is also expressed as the sum of two parts: isotropic part $\rho_{0}(r)$ and the anisotropic part $\rho_{2}(r)$

$$
\rho(r, \varphi)=\rho_{0}(r)-\cos (2 \varphi) \rho_{2}(r)
$$

where

$$
\left\{\begin{array}{l}
\rho_{0}(r)=\int_{0}^{\infty} S(K) J_{0}(K r) d K \\
\rho_{2}(r)=\int_{0}^{\infty} S(K) J_{2}(K r) \Delta(K) d K
\end{array}\right.
$$

$J_{n}$ is the $n$th order Bessel function of the first kind.

In this study, two typical omnidirectional Apel [19] and Elfouhaily spectra [17] are selected. The roughness parameters derived from the sea spectral components by using Equations (3) and (4) are input into the scattering model, AIEM, to be shown next.

\subsection{Advanced Integral Equation Model}

Numerical models and analytical models are two commonly used theoretical scattering models $[8,22]$. The former, based on the solution of Maxwell equations, can produce simulations with high accuracy. However, they are extremely computationally expensive and thus it is very difficult to analyze the scattering behaviors from large-scale sea surface due to the limited size of simulated surface profile (e.g., the maximal area size: $64 \lambda \times 64 \lambda, \lambda$ is the wavelength for L-band) [23]. In contrast, the analytical models, derived under reasonable assumptions and approximations, give a good balance between prediction accuracy and computational efficiency, which makes them easy and efficient to employ in various microwave remote sensing applications and theoretical studies [24]. The analytical models include the conventional models, e.g., KA and SPM, and the unifying models, e.g., TSM, Small Slope Approximation (SSA), IEM and its improved version, AIEM [25-32]. Figure 1 depicts the general geometry of electromagnetic wave scattering from rough sea surface. As an incident plane wave with spatial wavenumber $k$ propagates along the direction incident angle $\theta_{i}$ and azimuth angle $\phi_{i}$ and impinges onto a rough sea surface, the upwardly scattered field at scattering angle $\theta_{s}$ and azimuth 
angle $\phi_{s}$ is then observed by sensors in the upper half-space. The wave components projected onto three axes of the incident and scattering waves are given by:

$$
\begin{aligned}
& k_{i x}=k \sin \theta_{i} \cos \phi_{i} k_{i y}=k \sin \theta_{i} \sin \phi_{i} k_{i z}=k \cos \theta_{i} \\
& k_{s x}=k \sin \theta_{s} \cos \phi_{s} k_{s y}=k \sin \theta_{s} \sin \phi_{s} k_{s z}=k \cos \theta_{s}
\end{aligned}
$$

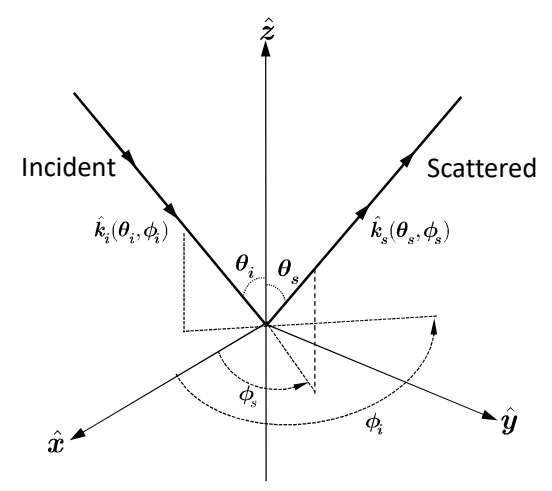

Figure 1. The geometry sketch of electromagnetic wave scattering from sea surface.

The AIEM, a widely used model [33,34], is selected as a working model for characterizing backscattering from multiscale sea surface. For self-containment, only the final expressions of single surface scattering, which is applicable to the rough surface with the RMS surface slope less than 0.4, is given in Equation (9). The model expression takes the following form:

$$
\sigma_{q p}^{0}=\frac{k}{2} \times \exp \left[-\sigma^{2}\left(k_{i z}^{2}+k_{s z}^{2}\right)\right] \sum_{n=1}^{\infty}\left|I_{q p}^{n}\right|^{2} \frac{\sigma^{2 n} \mathbf{W}^{(n)}(K, \phi)}{n !}
$$

where the roughness spectrum, $\mathbf{W}^{(n)}(K, \phi)$, is the Fourier transform of the $n$th power of the surface correlation function $\rho(r, \varphi)$ :

$$
\mathbf{W}^{(n)}(K, \phi)=\frac{1}{2 \pi} \int_{0}^{\infty} \int_{0}^{2 \pi} \rho^{n}(r, \varphi) \exp (j K r \cos (\varphi-\phi)) r d r d \varphi
$$

with

$$
\begin{gathered}
K=\sqrt{\left(k_{s x}-k_{i x}\right)^{2}+\left(k_{s y}-k_{i y}\right)^{2}} \\
\phi=\tan ^{-1}\left\{\left(k_{s x}-k_{i x}\right) /\left(k_{s y}-k_{i y}\right)\right\}
\end{gathered}
$$

The factor $\mathbf{I}_{q p}^{n}$ in Equation (9) is explicitly given by:

$$
\begin{aligned}
\mathbf{I}_{q p}^{n}=\left(k_{s z}+\right. & \left.k_{i z}\right)^{n} f_{q p} \exp \left(-\sigma^{2} k_{i z} k_{s z}\right) \\
+\frac{1}{4}\{ & F_{q p}^{+}\left(-k_{i x},-k_{i y}\right)\left(k_{s z}-k_{i z}\right)^{n} \times \exp \left[-\sigma^{2}\left(k_{i z}^{2}-k_{i z}\left(k_{s z}-k_{i z}\right)\right)\right] \\
& +F_{q p}^{-}\left(-k_{i x},-k_{i y}\right)\left(k_{s z}+k_{i z}\right)^{n} \times \exp \left[-\sigma^{2}\left(k_{i z}^{2}+k_{i z}\left(k_{s z}-k_{i z}\right)\right)\right] \\
& +F_{q p}^{+}\left(-k_{s x},-k_{s y}\right)\left(k_{i z}+k_{s z}\right)^{n} \times \exp \left[-\sigma^{2}\left(k_{i z}^{2}-k_{i z}\left(k_{s z}-k_{i z}\right)\right)\right] \\
& +F_{q p}^{-}\left(-k_{s x},-k_{s y}\right)\left(k_{i z}-k_{s z}\right)^{n} \times \exp \left[-\sigma^{2}\left(k_{i z}^{2}+k_{i z}\left(k_{s z}-k_{i z}\right)\right)\right] \\
& +G_{q p}^{+}\left(-k_{i x},-k_{i y}\right)\left(k_{s z}-k_{t i z}\right)^{n} \times \exp \left[-\sigma^{2}\left(k_{t i z}^{2}-k_{t i z}\left(k_{s z}-k_{i z}\right)\right)\right] \\
& +G_{q p}^{-}\left(-k_{i x},-k_{i y}\right)\left(k_{s z}+k_{t i z}\right)^{n} \times \exp \left[-\sigma^{2}\left(k_{t i z}^{2}+k_{t i z}\left(k_{s z}-k_{i z}\right)\right)\right] \\
& +G_{q p}^{+}\left(-k_{s x},-k_{s y}\right)\left(k_{i z}+k_{t s z}\right)^{n} \times \exp \left[-\sigma^{2}\left(k_{t s z}^{2}-k_{t s z}\left(k_{s z}-k_{i z}\right)\right)\right] \\
& \left.+G_{q p}^{-}\left(-k_{s x},-k_{s y}\right)\left(k_{i z}-k_{t s z}\right)^{n} \times \exp \left[-\sigma^{2}\left(k_{t s z}^{2}+k_{t s z}\left(k_{s z}-k_{i z}\right)\right)\right]\right\}
\end{aligned}
$$


Through Equations (9)-(12), the subscripts $q$ and $p$ denote the received and transmitted polarizations, respectively; $\sigma$ is the RMS height of the surface. The Kirchhoff field coefficient $f_{q p}$ and the complementary scattered fields propagating through the upward $(+)$ and downward $(-)$ directions in both the upper and lower medium $F( \pm), G( \pm)$ are explicitly given in references [32-35]. In this context, the parameter $K$ corresponds to a specific spectral component of ocean surface roughness spectrum model and is determined by incident frequency and radar observation geometry. Although we have given the general expression of this scattering model, the backward observation configuration is what we're interested in, due to the abundant radar measurements for assessing the performance of this study. For backscatter mode, the transmitter and receiver are colocated and the cross polarizations (i.e., $\mathrm{HV}$ and $\mathrm{VH}$ ) from the sea surface are much smaller than that of the like polarizations (i.e., $\mathrm{HH}$ and VV), due to the small RMS slope (generally less than 0.3 within the wind speed of $30 \mathrm{~m} / \mathrm{s}$ [17]), which are not shown in this paper.

\subsection{Validation Data}

In this paper, three types of validation data at L-, C-, and Ku-bands are adopted to examine the effective roughness for radar backscattering. The L-band validation data for different wind directions comes from the Aquarius instrument equipped with scatterometer operating at $1.26 \mathrm{GHz}$, which has three beams with incident angles 28.7, 37.8, and 45.6 degrees, respectively. Each beam acquires backscattering coefficients from the sea surface when the wind speeds are $3,5,8,10,12$, and $15 \mathrm{~m} / \mathrm{s}$. These radar data were usually adopted to verify numerical simulations $[36,37]$. At low wind speeds (3-8 $\mathrm{m} / \mathrm{s})$, the negative upwind-crosswind asymmetry (NUC) of radar backscattering coefficients from the sea surface at L-band was observed by the space-borne scatterometers and shown by Yueh et al. [36]. To explain the NUC at L-band, Du et al. [37] proposed an empirical angular spreading function (ASF) by matching with the L-band radar observations, especially for low wind speeds. Therefore, the empirical ASF was used to generate two-dimension directional spectrum for simulating backscattering coefficients versus wind directions.

The validation data in upwind direction for C-band $(5.3 \mathrm{GHz})$, incident angle $\theta_{i}=$ $\left\{18^{\circ}, 20^{\circ}, \cdots, 58^{\circ}\right\}$ and Ku-band $(14 \mathrm{GHz}), \theta_{i}=\left\{2^{\circ}, 4^{\circ}, \cdots, 60^{\circ}\right\}$ at wind speeds $U_{10}=5,10$, and $15 \mathrm{~m} / \mathrm{s}$, comes from empirical scattering models C-band geophysical model function (CMOD7) [38] and NASA scatterometer (NSCAT-4) [39] derived from extensive radar measurements, respectively.

\section{Scattering Source of Backscattering from Multiscale Sea Surface}

For a single-scale rough surface, the sources of surface scattering for the classical geometric optics, Kirchhoff, and small perturbation or Bragg scattering models are the surface slope distribution, reflection and diffraction, and surface spectrum evaluated at Bragg wavenumbers, respectively [9]. They are valid, respectively, in the high-frequency limit, high-frequency region, and low-frequency region. Because the AIEM model integrates the three models and includes them as special cases, the scattering source for AIEM in Equation (9) is the all orders of surface spectrum $\mathbf{W}^{(n)}(K, \phi)$ evaluated at the specific surface spectral component, $K=2 k \sin \theta_{i}$ (hereafter, called the effective wavenumber).

For multiscale rough sea surface, it is instructive to know that only a part of roughness scales (effective roughness) are responsible for backscattering at a given view angle and exploring frequency [11]. The contributions to backscattering by the other roughness do not add up coherently along the backscattering direction and are filtered by effective sensing wavelength $\lambda_{e}$, determined by the incident wavelength and incident angle, i.e., $\lambda_{e}=\lambda /\left(2 \sin \theta_{i}\right)[9,40]$, which is called "wavelength filtering effect" [9]. Therefore, the backscattering source for radar backscattering from multiscale sea surface is also the all orders of surface spectrum $\mathbf{W}^{(n)}(K, \phi)$ which attaches to a specific roughness determined by incident angle and frequency (i.e., effective roughness) [9]. 


\subsection{Various Roughness Derived from Sea Spectrum}

Different scales of roughness riding on sea surface can also be calculated by different part of sea spectral components, from which the effective roughness can be derived. Roughness scales responsible for radar scattering are usually characterized quantitatively by the correlation function and the surface RMS height. For illustration, let's take L-band $(28 \mathrm{rad} / \mathrm{m})$ as an example. The correlation functions and roughness parameters (RMS height and correlation length) are computed by different spectral components $k_{e} \in[0.1 * k, \infty),[0.05 * k, \infty)$, and $(0, \infty)$ based on Apel spectrum. The corresponding roughness parameters along the upwind- and crosswind-directions at the wind speeds of $10 \mathrm{~m} / \mathrm{s}$ and $15 \mathrm{~m} / \mathrm{s}$ are shown in Figure 2.

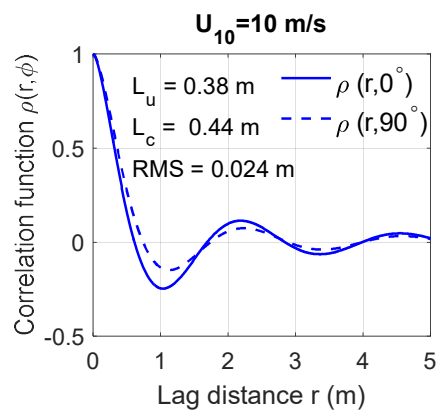

(a)

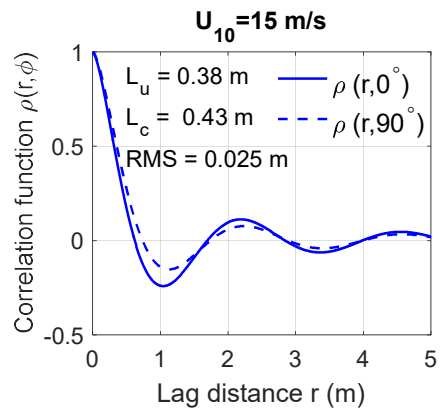

(b)

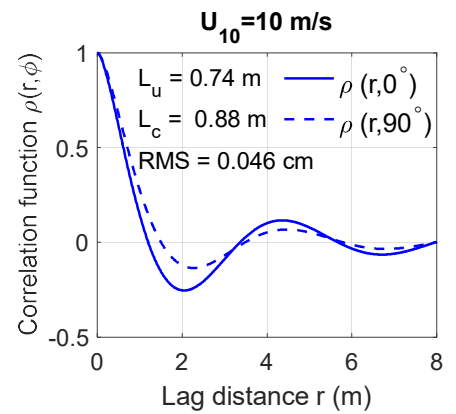

(c)

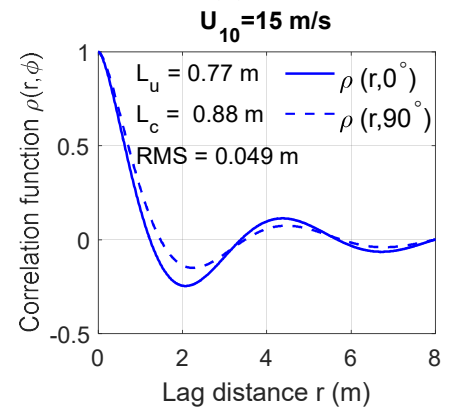

(d)

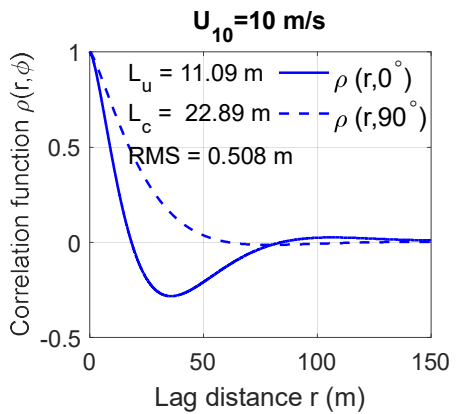

(e)

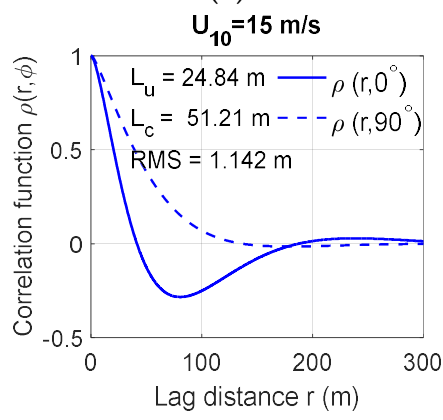

(f)

Figure 2. The normalized correlation functions and roughness parameters (RMS height and correlation length $\mathrm{Lu}, \mathrm{Lc}$ in upwind and crosswind directions) generated by different wave spectral components ke based on Apel spectrum model at L-band $(1.26 \mathrm{GHz})$ for wind speeds of $10 \mathrm{~m} / \mathrm{s}$ and $15 \mathrm{~m} / \mathrm{s}$, respectively. (a)-(b) $k_{e} \in\left[0.1^{*} k, \infty\right)$; (c)-(d) $k_{e} \in\left[0.05^{*} k, \infty\right) ;(\mathbf{e})-(\mathbf{f}) k_{e} \in(0, \infty)$ ).

It can be seen from Figure 2 that the normalized correlation functions generated by high-wavenumber spectral components (Figure $2 \mathrm{a}, \mathrm{b}$ ) are more oscillating with a decreasing amplitude and the RMS height and correlation lengths in both upwind- and crosswind-directions are also small, which is in accord with that of short wind wave fields derived from the along-wind surface wave profiles, shown in the Figure 13 of [41]. With the addition of low-wavenumber spectral components, the roughness increases. When all the spectral components in sea spectrum are included to account for the roughness (Figure 2e,f), the corresponding scatterer on sea surface is very large with a tens of meters of correlation length and tens of centimeters or even one meter in RMS height. Meanwhile, the oscillation of autocorrelation function of large scale scatterer also flattens out and the roughness becomes larger as the wind speed increases (i.e., satisfying the law of KA model). At the same time, for the case of Figure 2a-d the scatterer becomes rougher with a smaller correlation length and larger RMS height with the increase of wind speed (i.e., satisfying the law of the SPM model). 
3.2. Variation of Backscattering Sources $W^{(n)}$ Attaching to a Roughness with the Effective Wavenumber K and Number of Terms $n$

In this section we shall consider the variation of the backscattering source $W^{(n)}$ with effective wavenumber $K$ attaching to the largest roughness (Figure $2 \mathrm{e}, \mathrm{f}), k_{e} \in(0, \infty)$ ), taken with an example in the upwind direction when the $n$-value varies from 1 to 16 with a step of 3 . In backscatter mode, the effective wavenumber $K$ is equal to $2 k \sin \theta_{i}$. Since $0^{\circ} \leq \theta_{i}<90^{\circ}$, it follows that $0 \leq K / k<2$. Hence, only the surface spectral components within the range of $[0,2 k)$ are responsible for backscattering [9]. In order to show the variation of $W^{(n)}(K, \phi)$ along the upwind direction $\left(\phi=0^{\circ}\right)$ with the effective wavenumber $K$ and $n$, the numerical illustrations are shown in Figure 3.

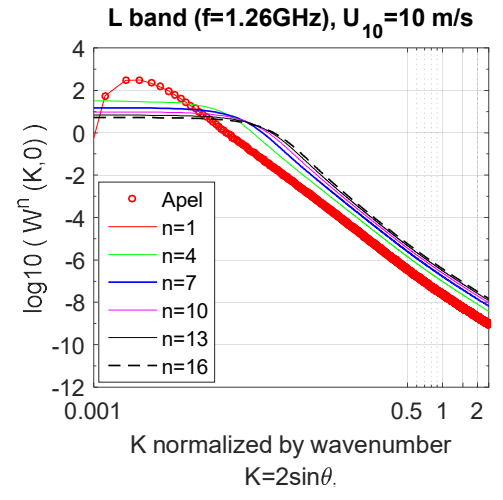

(a)

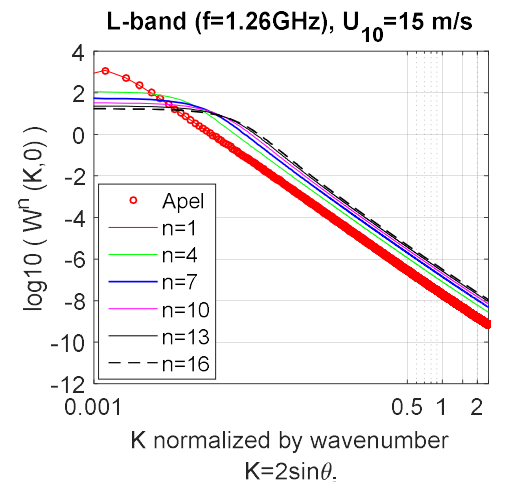

(b)

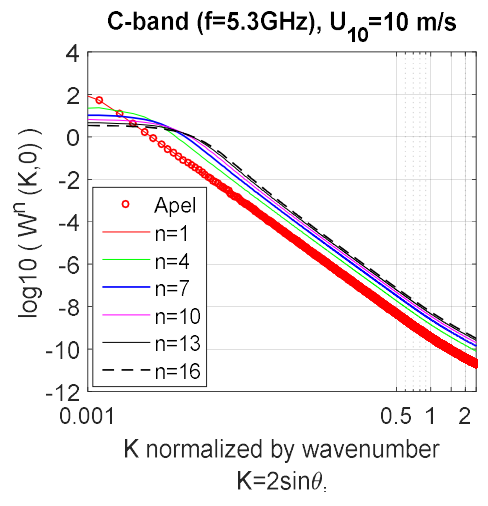

(c)

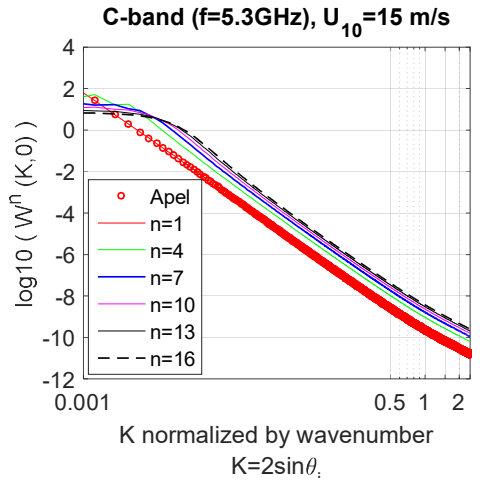

(d)

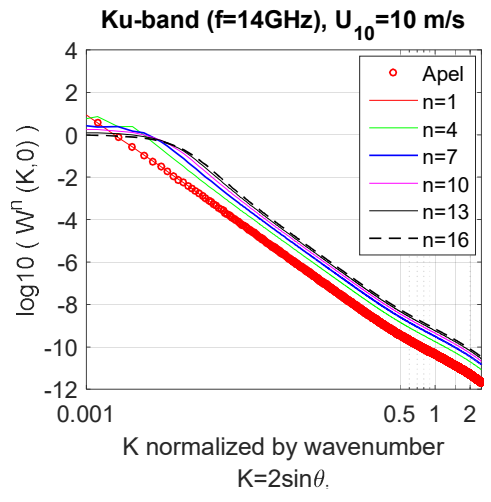

(e)

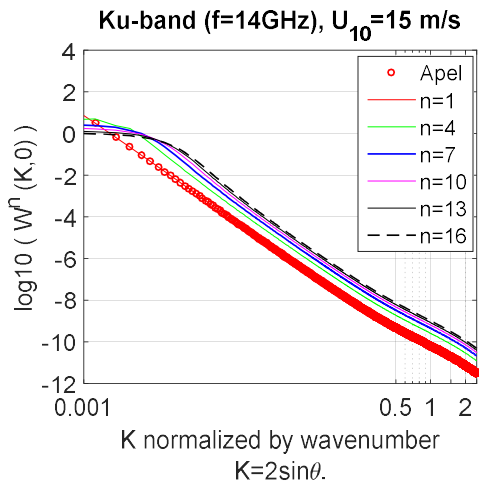

(f)

Figure 3. The variation of the relative importance of $W^{(n)}$ in the upwind direction with effective wavenumber $K$ normalized by exploring wavenumber (L-, C-, and Ku-bands) and $n$ at the wind speeds of $10 \mathrm{~m} / \mathrm{s}$ and $15 \mathrm{~m} / \mathrm{s}$, respectively. (a)-(b) $k_{e} \in\left[0.1^{*} k, \infty\right)$; (c)-(d) $k_{e} \in\left[0.05^{*} k, \infty\right) ;(\mathbf{e})-(\mathbf{f}) k_{e} \in\left[0.0005^{*} k, \infty\right)$.

For each subplot in Figure 3, there is a transition region of the relative importance of all orders of scatter source $W^{(n)}$ within the range of effective wavenumber $K$. Over the low-wavenumber region (near the nadir), the level becomes higher as the $n$-value increases, but it is inversed at a high-wavenumber regime (at medium and large incident angles). However, the increasing $n$-value does not lead to the monotonous increase in level over the transition region. In addition, the transition region shifts toward low-wavenumber area with the increase of exploring frequency and wind speed and also broadens with the increase of $n$-value. The $W^{(1)}$ is in accord with the original Apel sea spectrum denoted by the symbol "Apel" in the legend, indicating the relationship of surface correlation function and its surface spectrum by Fourier Transform. Besides, these variation trends along the crosswind direction are similar to that in upwind direction but slightly lower in level, which are not shown in this paper. 


\section{Effective Roughness Scales for Radar Backscattering}

As mentioned above, the idea that only an effective roughness scale is responsible for backscattering at a given frequency and incident angle is the most fundamental concept to understand the backscattering from multiscale rough sea surfaces. The effective roughness depends on frequency and incident angle [9]. For example, at centimeter wavelengths (L-, C-, and $\mathrm{Ku}$-bands) and medium incident angles the meter-size roughness scales are not responsible for radar backscattering from a multiscale rough surface, and locally the large undulating surface is now serving as a reference plane for scattering. In this section, the variations of backscattering simulations with different roughness scales derived from different sea spectrum components are firstly analyzed. Then the effective roughness responsible for radar backscattering from sea surface were determined by matching the simulations with the validation data from radar measurements and empirical models.

\subsection{Variation of Backscattering Simulations versus Different Roughness}

In order to determine the effective roughness responsible for radar backscattering, we first need to construct various roughness using different sea spectrum components as illustrated in Section 3.1. Because the scatter source responsible for backscattering is the all order of roughness spectrum $W^{(n)}(K)$ evaluated at effective wavenumber $K$, the range of spectral components used for generating roughness should contain the specific surface spectral component. Therefore, the lower limit $k_{L}$ of the range of sea spectral components $\left[k_{L}, \infty\right)$ should be related to $K$. In this paper, a simple linear relationship $k_{L}=m \times K$ is assumed to make an attempt to investigate the effective roughness on multiscale sea surface. Here, $m$ is referred to as the intercept parameter.

In order to explore the backscattering behaviors versus roughness determined by spectrum components $[m \times K, \infty)$, several simulation results at L-band, taken an example, for different roughness scales and incident angles along different wind directions based on Apel spectrum are analyzed when the wind speed is $10 \mathrm{~m} / \mathrm{s}$. The intercept parameter $m=0.1,0.05$, and 0.01 and the incident angles are 10,30, and 50 degrees. Other parameters related to the backscattering simulation are sea surface temperature and sea water salinity, which are set as $20^{\circ} \mathrm{C}$ and $35 \%$, respectively. Based on the two parameters, the relative permittivity is computed from the Debye equation [42]. These simulations are plotted in Figure 4. 


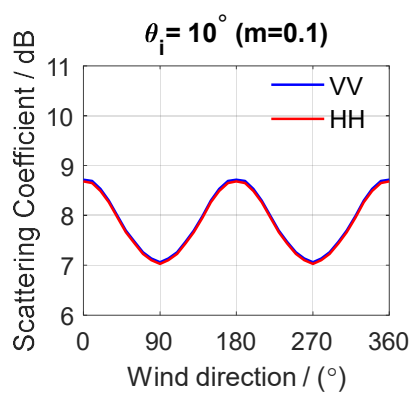

(a)

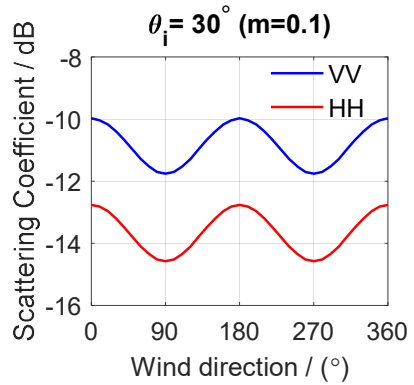

(b)

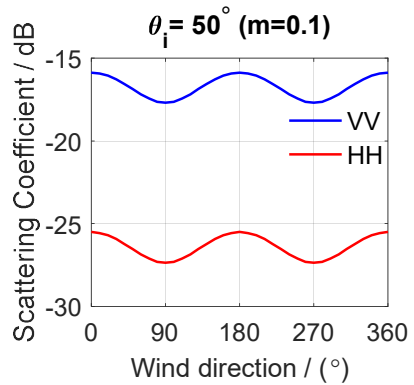

(c)

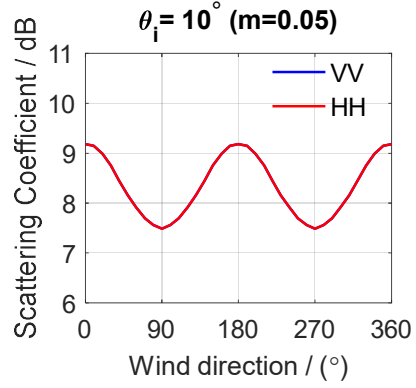

(d)

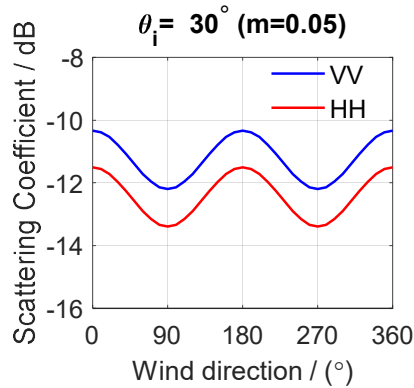

(e)

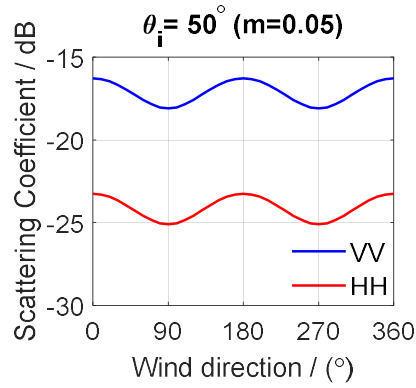

(f)

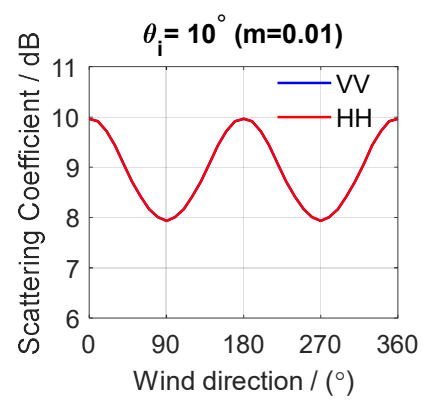

(g)

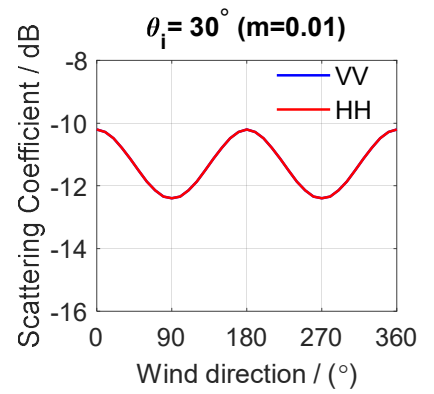

(h)

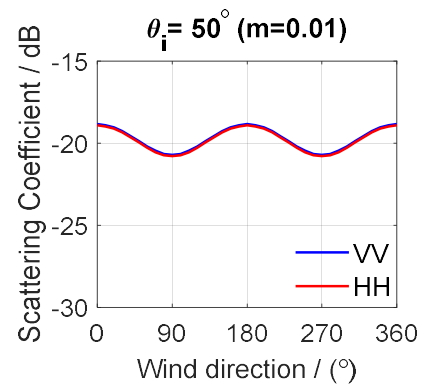

(i)

Figure 4. The theoretical backscattering behaviors relative to wind direction versus $m$ at wind speed of $10 \mathrm{~m} / \mathrm{s}$ with incident angles of 10,30 , and 50 degrees. (a) - (c) $m=0.1 ;(\mathbf{d})-(\mathbf{f}) m=0.05 ;$ (g) $-(\mathbf{i}) m=0.01$.

It can be clearly seen from Figure 4 that for small $m$-value (0.01) there is no difference between VVand $\mathrm{HH}$-polarizations regardless of incident angle. This is because for this situation the behavior of backscattering from sea surface is dominated by large roughness relative to the effective wavelength where AIEM model reduces to KA limits (no polarization difference). When $m=0.1$ and 0.05 (small and medium roughness), the levels of $\mathrm{HH}$ - and VV-polarized backscattering coefficient decrease with the increase of incident angle and the difference between them also gradually increases, which is more evident for larger $m$-value. Besides, the two polarizations shift toward each other as the $m$-value decreases but the $\mathrm{HH}$ polarization is more sensitive.

\subsection{Effective Roughness}

Based on the effect of $m$-value on behaviors of backscattering coefficients, the $m$-value is determined by matching the simulated backscattering coefficients to experimental measurements. Thus, the effective roughness generated by the effective spectral components $[m \times K, \infty)$ is also determined.

For L-band, when all the validation data are matched with the backscattering simulations, we found that the $m$-value is about 0.05 . Although the $m$-value is a constant, the effective wavenumber $K=2 k \sin \theta_{i}$ varies with the incident angle $\theta_{i}$ and exploring wavenumber $k$, which further leads to a change in spectral components $[m \times K, \infty)$ for constructing the effective roughness. The comparisons with Aquarius data along various wind directions at several combinations of incident angle and wind 
speed are shown in Figure 5. The corresponding roughness parameters for L-band radar backscattering and the effective wavelength are listed in Table 1.

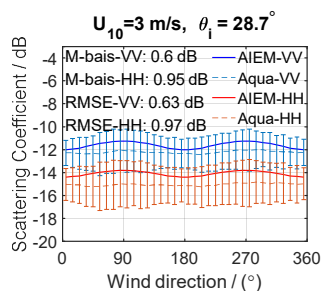

(a1)

$\mathrm{U}_{10}=5 \mathrm{~m} / \mathrm{s}, \theta_{\mathrm{i}}=28.7^{\circ}$

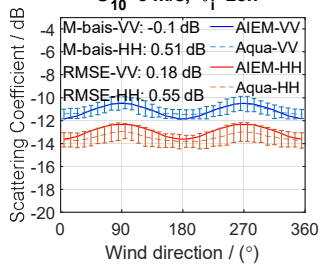

(a2)

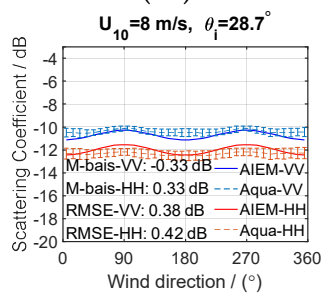

(a3)

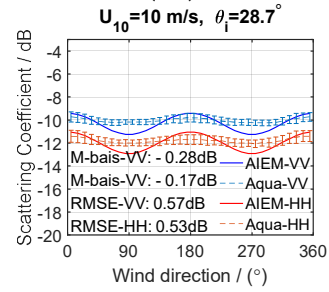

(a4)

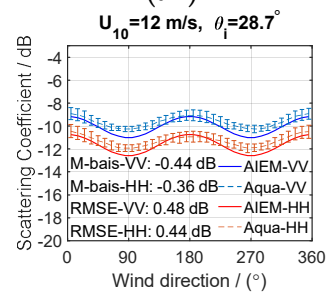

(a5)

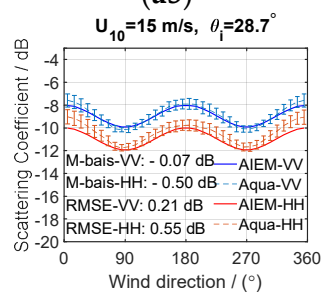

(a6)

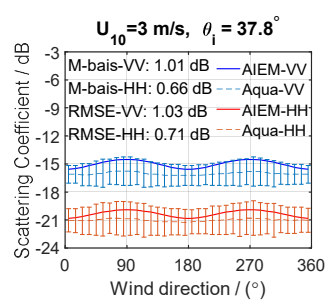

(b1)

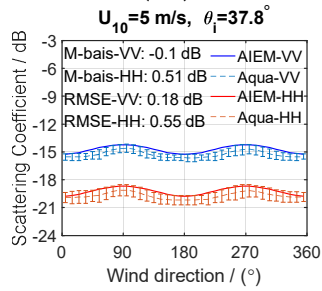

(b2)

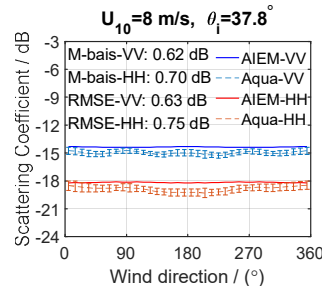

(b3)

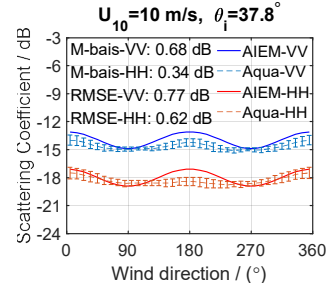

(b4)

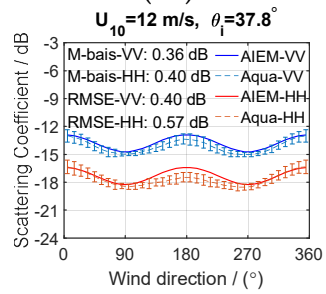

(b5)

$\mathrm{U}_{10}=15 \mathrm{~m} / \mathrm{s}, \theta_{\mathrm{i}}=37.8^{\circ}$

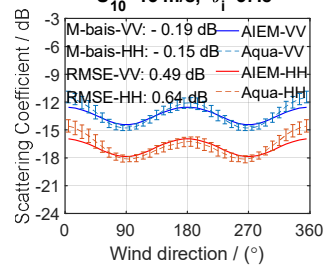

(b6)

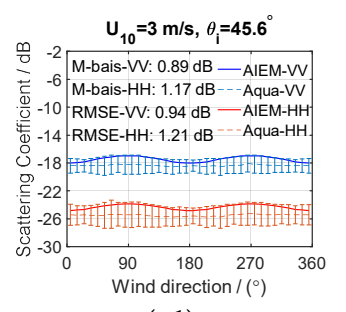

(c1)

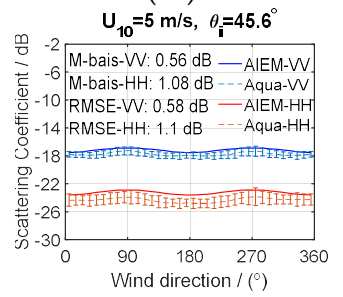

(c2)

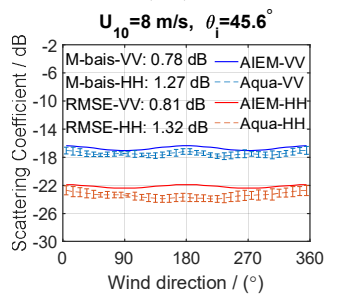

(c3)

$U_{10}=10 \mathrm{~m} / \mathrm{s}, \theta_{\mathrm{i}}=45.6^{\circ}$

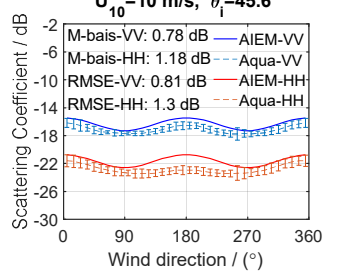

(c4)

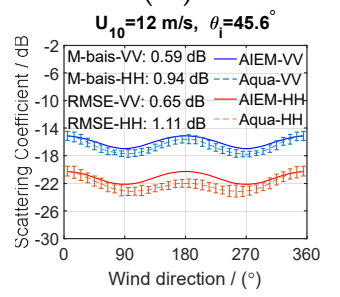

(c5)

$U_{10}=15 \mathrm{~m} / \mathrm{s}, \theta_{\mathrm{i}}=45.6$

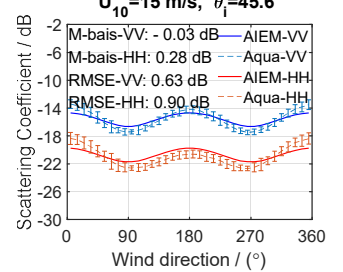

(c6)

Figure 5. Comparison of simulated backscattering based on AIEM and Apel sea spectrum and Aquarius radar data at L-band for incidence angles of 28.7, 37.8, and 45.6 degrees and wind speeds of 3, 5, 8, 10, 12, and $15 \mathrm{~m} / \mathrm{s}$, respectively. (a1)-(c1) $3 \mathrm{~m} / \mathrm{s}$; (a2)-(c2) $5 \mathrm{~m} / \mathrm{s}$; (a3)-(c3) $8 \mathrm{~m} / \mathrm{s}$; (a4)-(c4) $10 \mathrm{~m} / \mathrm{s}$; (a5)-(c5) $12 \mathrm{~m} / \mathrm{s}$; (a6)-(c6) $15 \mathrm{~m} / \mathrm{s}$. 
Table 1. The effective roughness parameters corresponding to Figure 5 in the upwind direction (wind direction $=0^{\circ}$ ) at incident angles of $28.7,37.8$, and 45.6 degrees with wind speeds of $3,5,8,10,12$, and $15 \mathrm{~m} / \mathrm{s}$.

\begin{tabular}{|c|c|c|c|c|}
\hline Incident Angles $\theta_{i}\left({ }^{\circ}\right)$ & $\begin{array}{l}\text { Wind Speed } U_{10} \\
(\mathrm{~m} / \mathrm{s})\end{array}$ & $\begin{array}{l}\text { RMS Height } \sigma \\
\text { (cm) }\end{array}$ & $\begin{array}{c}\text { Correlation } \\
\text { Length } C_{1}(\mathrm{~cm})\end{array}$ & $\begin{array}{c}\text { Effective Wavelength } \\
\lambda \mathrm{e}(\mathrm{cm})\end{array}$ \\
\hline \multirow{6}{*}{28.7} & 3 & 3.1 & 63 & \multirow{6}{*}{24.7} \\
\hline & 5 & 3.8 & 73 & \\
\hline & 8 & 4.5 & 78 & \\
\hline & 10 & 4.8 & 78 & \\
\hline & 12 & 4.9 & 78 & \\
\hline & 15 & 5.1 & 78 & \\
\hline \multirow{6}{*}{37.8} & 3 & 2.6 & 48 & \multirow{6}{*}{19.4} \\
\hline & 5 & 3.2 & 58 & \\
\hline & 8 & 3.7 & 63 & \\
\hline & 10 & 3.8 & 63 & \\
\hline & 12 & 3.9 & 63 & \\
\hline & 15 & 4.1 & 63 & \\
\hline \multirow{6}{*}{45.6} & 3 & 2.3 & 44 & \multirow{6}{*}{16.7} \\
\hline & 5 & 2.8 & 54 & \\
\hline & 8 & 3.2 & 54 & \\
\hline & 10 & 3.3 & 54 & \\
\hline & 12 & 3.4 & 54 & \\
\hline & 15 & 3.6 & 54 & \\
\hline
\end{tabular}

Similarly, for C- and Ku-bands, when the intercept parameter $m$ is set to be 0.05 , the simulated backscattering coefficients versus incident angles based on Apel spectrum for 5, 10, and $15 \mathrm{~m} / \mathrm{s}$ also are in good agreement with the empirical CMOD7 (no HH polarization) and NSCAT-4 models except for Ku-band at $10 \mathrm{~m} / \mathrm{s}$ and $15 \mathrm{~m} / \mathrm{s}$. This indicates that the $m$-value may be an intrinsic property for calculating the effective roughness for radar backscattering. The discrepancy of Ku-band at $10 \mathrm{~m} / \mathrm{s}$ and $15 \mathrm{~m} / \mathrm{s}$ will be further analyzed in the discussion section. The related comparisons with the validation data were shown in Figures $6 \mathrm{a}-\mathrm{c}$ and $7 \mathrm{a}-\mathrm{c}$, respectively. Then the corresponding roughness parameters RMS height, correlation length, and their ratio were also calculated and exhibited in Figures $6 \mathrm{~d}-\mathrm{f}$ and $7 \mathrm{~d}-\mathrm{f}$, respectively.

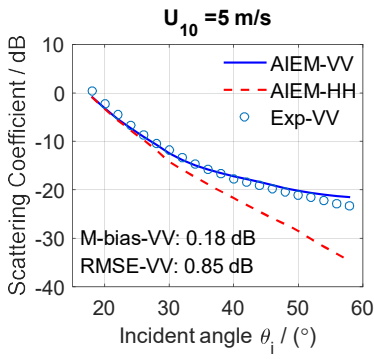

(a)

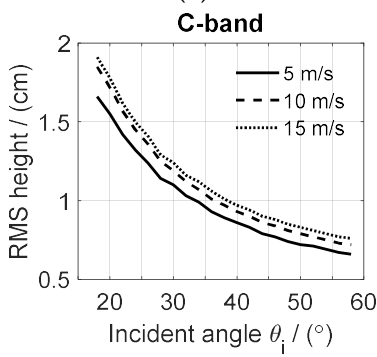

(d)

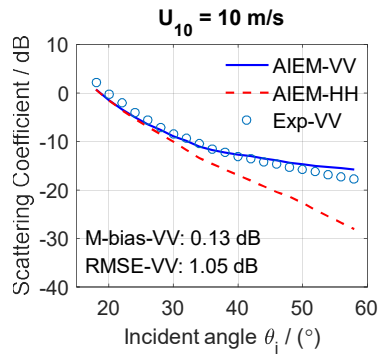

(b)

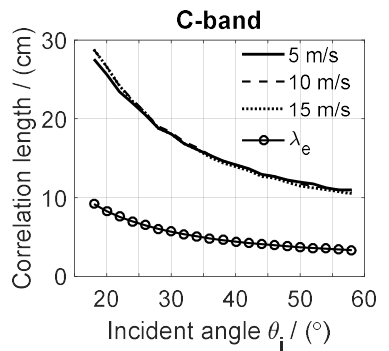

(e)

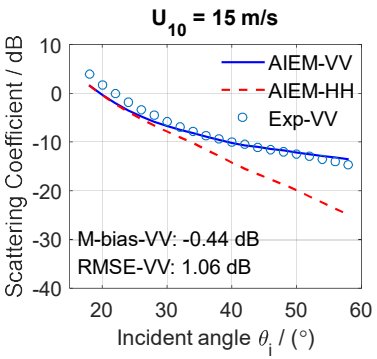

(c)

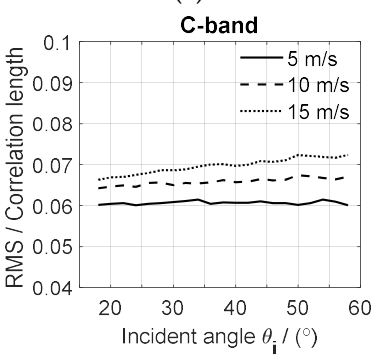

(f)

Figure 6. Comparisons of the simulated backscattering based on Apel spectrum for C-band with the CMOD7 data (no $\mathrm{HH}$ polarization) at the wind speeds of $5 \mathrm{~m} / \mathrm{s}, 10 \mathrm{~m} / \mathrm{s}$, and $15 \mathrm{~m} / \mathrm{s}$ (the first row: (a) $5 \mathrm{~m} / \mathrm{s}$; (b) $10 \mathrm{~m} / \mathrm{s}$; (c) $15 \mathrm{~m} / \mathrm{s}$ ) and the corresponding roughness parameters (the second row: (d) RMS height, (e) correlation length, and (f) the ratio of RMS and correlation length). 


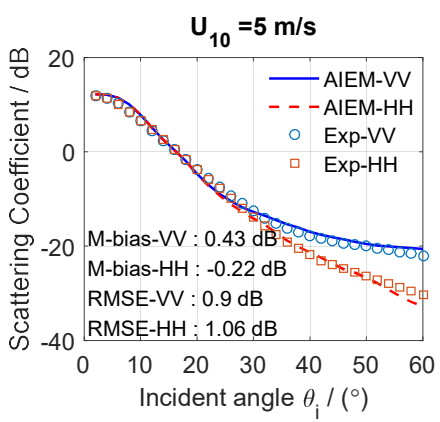

(a)

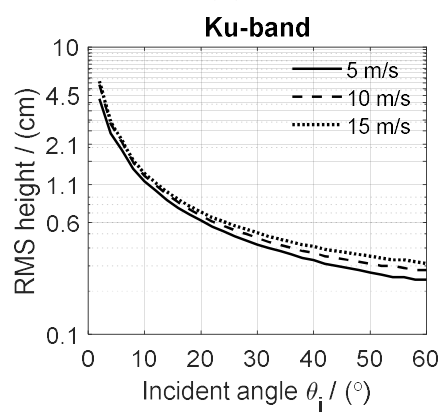

(d)

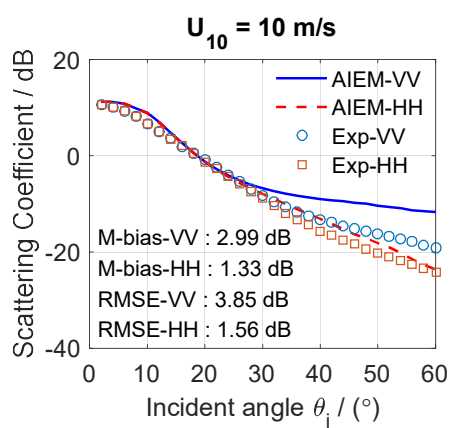

(b)

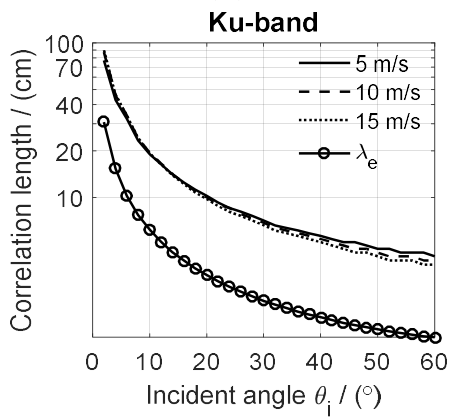

(e)

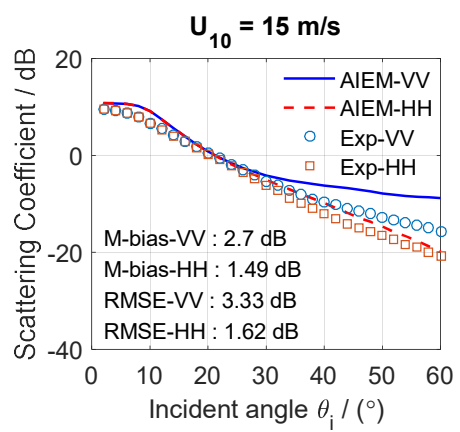

(c)

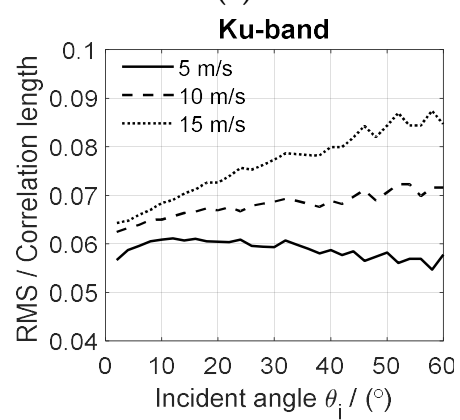

(f)

Figure 7. Comparisons of the simulated backscattering based on Apel spectrum for Ku-band with NSCAT-4 model at the wind speeds of (a) $5 \mathrm{~m} / \mathrm{s}$, (b) $10 \mathrm{~m} / \mathrm{s}$, and (c) $15 \mathrm{~m} / \mathrm{s}$; and the corresponding roughness parameters (d) RMS height, (e) correlation length and effective wavelength $\left(\lambda_{\mathrm{e}}\right)$, and (f) the ratio of RMS and correlation length.

\section{Discussion}

\subsection{Analysis of Effective Roughness}

In Figure 5, when the $m$-value is set to 0.05 , the simulated backscattering coefficients based on AIEM model and the Apel spectrum for L-band are overall in good agreement with the Aquarius radar measurements with the error of around $1 \mathrm{~dB}$ for $\mathrm{HH}$ - and $\mathrm{VV}$ - polarizations for the incidence angles of 28.7, 37.8, and 45.6 degrees and the wind speeds of 3, 5, 8, 10, 12, and $15 \mathrm{~m} / \mathrm{s}$. Moreover, the $m$-value is independent of incident angles, wind directions, and wind speeds. Based on this, the corresponding roughness parameters (RMS height and correlation length) responsible for radar backscattering are calculated and are shown in Table 1. As incidence angle increases, the effective spectral components reduce and the effective roughness for radar backscattering gradually becomes smaller (i.e., the decreasing RMS height and correlation length). Besides this, the correlation length has little change but the RMS height becomes larger as the wind speed increases, indicating a rougher surface (scatterer). In addition, the correlation length for the three incident angles has the same variation trend with effective wavelength and can be described by an approximate linear relation:

$$
C_{l}=3.2 \times \lambda_{e}
$$

For C-band, the same $m$-value to that for L-band also makes the simulated VV-polarized backscattering coefficients well close to the empirical CMOD7 model with the error of mean bias and root mean square error (RMSE) within $1 \mathrm{~dB}$ for the three wind speeds. In Figure $6 \mathrm{~d}$,e, both the RMS height and correlation length decrease with the increase of incidence angles, suggesting that the roughness responsible for radar backscattering from sea surface becomes small with the increase of incident angles. Similar to the results of L band, the RMS height varies a great deal with wind speeds but the correlation length does not vary much. Likewise, the correlation length and the effective 
wavelength also have a high correlation as the incident angle changes (Figure 6e). The relationship can be also expressed by Equation (14). Furthermore, the ratio of RMS height and correlation length in Figure $6 \mathrm{f}$ is very small and increases slightly versus increasing incident angles, which indicates the single scattering is dominant and meets the requirements of the single-scattering AIEM model.

As for Ku-band, the simulated backscattering versus incident angles based on Apel spectrum and the same intercept parameter (i.e., $m=0.05$ ) as L- and C-bands are consistent better with the NSCAT-4 model at small wind speed (i.e., $5 \mathrm{~m} / \mathrm{s}$, see Figure $7 \mathrm{a}$ ). Meanwhile at wind speeds of $10 \mathrm{~m} / \mathrm{s}$ (Figure $7 \mathrm{~b}$ ) and $15 \mathrm{~m} / \mathrm{s}$ (Figure $7 \mathrm{c}$ ), the simulations are in agreement with the experimental data at small incidence angles $\left(\theta_{i} \leq 30^{\circ}\right)$, but substantially biased at medium to large incidence, especially for VV-polarization. At a small incident angle, the effective wavelength is very large and the effective roughness dominating the backscatter signal return is also large, which causes the scattering behavior to follow the KA with no difference between $\mathrm{HH}$ and VV polarizations. As the incident angle increases, the effective wavelength also decreases. Thus, the effective roughness becomes small with the increasing incident angle. Similarly, the correlation length and effective wavelength obey the relationship of Equation (14). However, the inconsistency of the simulated backscattering at large incident angles and high wind speeds of $10 \mathrm{~m} / \mathrm{s}$ and $15 \mathrm{~m} / \mathrm{s}$ may not be due to the $m$-value. This is because when the $m$-value becomes larger or smaller than $0.05, \mathrm{VV}$ - and $\mathrm{HH}$-polarizations will move away or towards each other, at medium and large incident angles, respectively (see Figure 4 in Section 4.1). Therefore, the overestimation of VV- polarization mentioned above may be given rise by the Apel spectrum itself and this behavior also was reported in reference [19]. This perhaps is because that the high-frequency spectral components in Apel model is overestimated, which has been proved by Elfouhaily et al. [17] through assessing the RMS slope, mainly determined by the high-frequency components, with the measurements by Cox and Munk [43]. However, the high-wavenumber components (capillary waves) are important for microwave backscattering especially for high frequency bands. To examine this idea, it is desirable to test the Elfouhaily spectrum in simulated backscattering, which will be shown in the following section.

\subsection{Effect of Sea Spectrum Model on Backscattering Simulations}

To examine the effect of the sea spectrum model on backscattering simulations, the Elfouhaily spectrum was adopted to simulate backscattering from the sea surface in this section. The simulations for the wind speeds of $10 \mathrm{~m} / \mathrm{s}$ and $15 \mathrm{~m} / \mathrm{s}$ based on Elfouhaily spectrum and the same $m$-value (i.e., $m=0.05)$ are shown in Figure 8a,e, respectively.

In Figure $8 \mathrm{a}$,e, the simulated VV polarization backscattering coefficients are closer to the experimental data, compared to simulated $\mathrm{HH}$ polarization, which is underestimated. Comparing with Figure $7 \mathrm{~b}, \mathrm{c}$, it can been seen that the simulated backscattering based on Apel spectrum are higher than those based on Elfouhaily spectrum except for at incident angles within 10 degrees where the backscattering behaviors are dominated by the RMS slope, i.e., following the GO model. Over the intermediate incidence range, the effective roughness based on the Apel spectrum is much rougher than that based on the Elfouhaily spectrum (Figure $8 \mathrm{~b}, \mathrm{f}$ ). This may be because the higher spectral components in Apel spectrum are larger than that in Elfouhaily spectrum over the high wavenumber region (Figure $8 \mathrm{c}, \mathrm{g}$ ). Thus, the simulated backscattering based on the Apel spectrum is higher than that based on the Elfouhaily spectrum at large incident angles. To further verify the deviation for Ku-band at high wind speeds impacted by sea spectrum itself, the simulated scattering coefficients based on the Elfouhaily spectrum by the first order SSA-1 scattering model [44] are calculated and shown in Figure $8 \mathrm{~d}, \mathrm{~h}$. They are in better agreement with each other except for a slight deviation in the incidence region ranging from 10 to 30 degrees and the results simulated by SSA- 1 are also consistent with (the Figure 3 of [45]). 


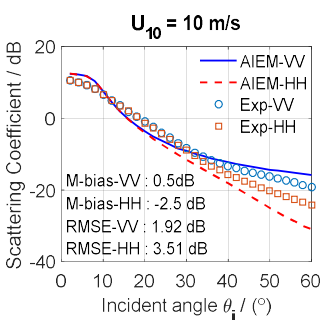

(a)

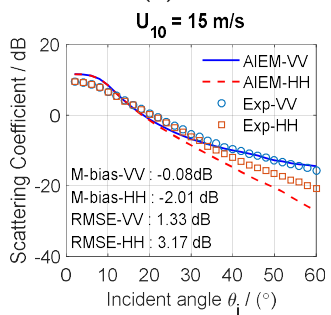

(e)

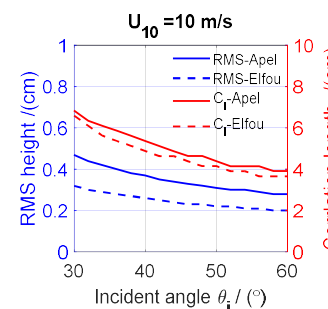

(b)

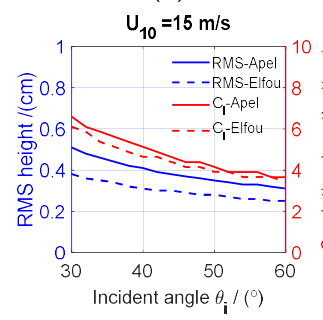

(f)

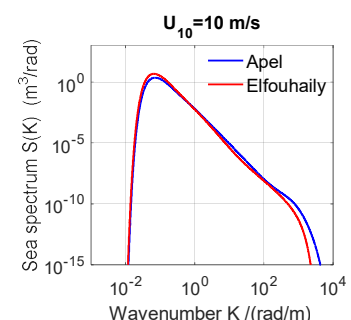

(c)

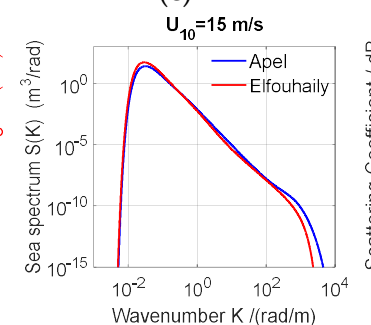

(g)

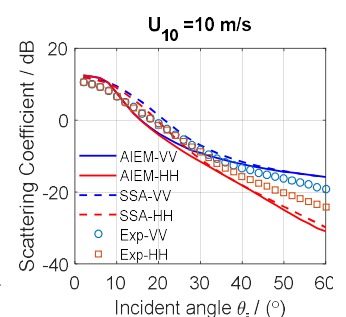

(d)

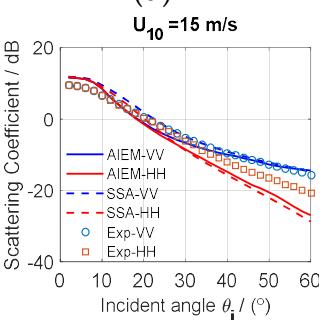

(h)

Figure 8. Comparisons of simulated backscattering, predicted by AIEM and SSA-1 model, versus incident angles based on Elfouhaily spectrum for Ku band with NSCAT-4 model at the wind speeds of $10 \mathrm{~m} / \mathrm{s}(\mathbf{a}),(\mathbf{d})$ and $15 \mathrm{~m} / \mathrm{s}(\mathbf{e}),(\mathbf{h})$; and the corresponding effective roughness parameters (b), (f) and spectrum curve (c), (g).

For the simulations for VV-polarization at C-band based on Elfouhaily spectrum, they were verified with the empirical model in reference [45] and the simulated results based on SSA-1 and Elfouhaily spectrum coincide with the simulations using the Apel spectrum in this paper. In addition, in order to investigate the effect of sea spectrum on the simulations at low frequency, the simulation results at L-band in the upwind direction based on the Elfouhaily and Apel spectra using the same $m$-value (i.e., 0.05 ) were also compared in Figure 9.

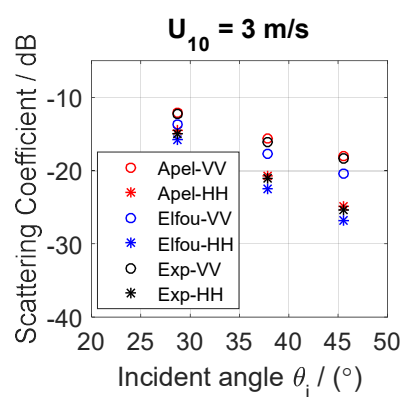

(a)

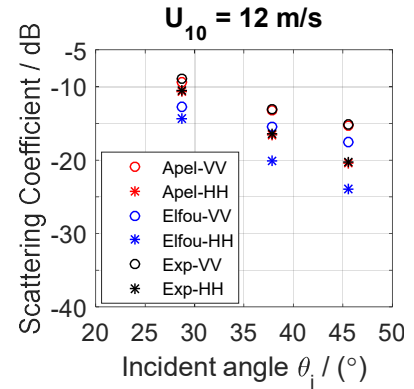

(d)

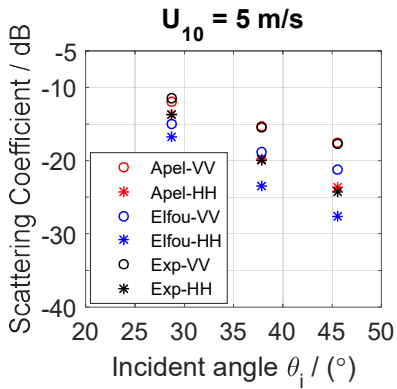

(b)

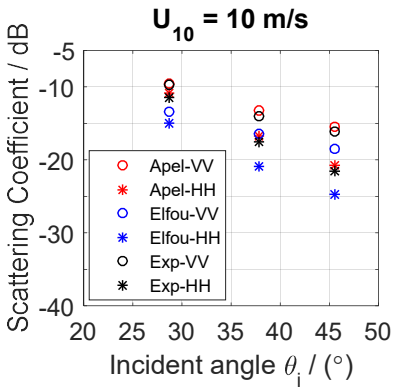

(e)

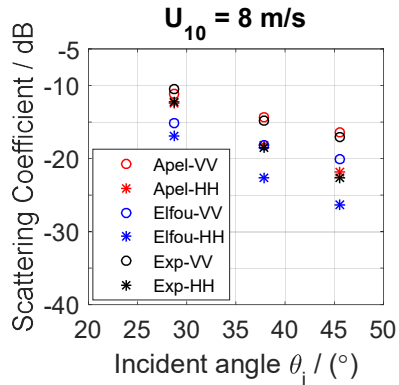

(c)

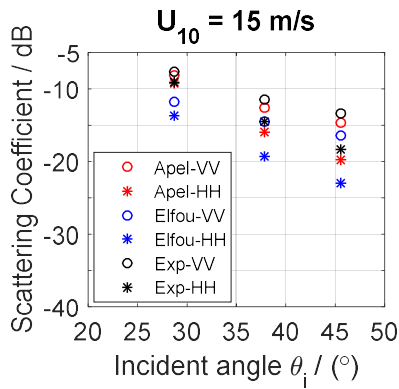

(f)

Figure 9. Comparisons of simulated backscattering based on Apel and Elfouhaily spectra with Aquarius data in the upwind direction at incident angles of 28.7,37.8, and 45.6 degrees for L-band at wind speeds of $3,5,8,10,12$, and $15 \mathrm{~m} / \mathrm{s}$, respectively. (a) $3 \mathrm{~m} / \mathrm{s}$; (b) $5 \mathrm{~m} / \mathrm{s}$; (c) $8 \mathrm{~m} / \mathrm{s}$; (d) $10 \mathrm{~m} / \mathrm{s}$; (e) $12 \mathrm{~m} / \mathrm{s}$; (f) $15 \mathrm{~m} / \mathrm{s}$. 
It is clearly seen from Figure 9 that the simulations (red symbols) based on the Apel spectrum are closer to the radar measurements (black symbols) especially for 3-12 m/s while the simulations (blue symbols) based on the Elfouhaily spectrum are underestimated significantly compared with the measured data. Therefore, for the low-frequency L-band, the Apel spectrum is more suitable for simulating radar backscattering. This may be because the related parameters used for building the Apel spectrum model are determined by radar data, making the low-frequency spectral components (gravity and capillary-gravity waves) more accurate [19]. While, for Elfouhaily spectrum, only the measurements in the tank were involved but the RMS slope of the spectrum, mainly determined by the high-frequency spectral components, is more consistent with the Cox and Munk measurements [43]. This perhaps explains why the Elfouhaily spectrum is more applicable to high-frequency Ku-band at high wind speed (e.g., greater than $10 \mathrm{~m} / \mathrm{s}$ ) and large incident angles (e.g., greater than 30 degrees). Overall, the roughness generated by using the spectral components from the classical Apel and Elfouhaily spectra is responsible for radar backscattering when the $m$-value is 0.05 , which is independent of incident angles, wind speeds, and radar frequencies.

In addition, it should be noted that the incidence angles and wind speeds are both moderate here and the predictions at large incidence angles (e.g., greater than 70 degrees) and high wind speeds (e.g., greater than $25 \mathrm{~m} / \mathrm{s}$ ) are challenging for general rough surface scattering models. This is because at high wind speed, the sea spectrum model (e.g., Apel and Elfouhaily spectra) is less accurate for describing the rough characteristics of the sea surface, while the break waves will also change the scattering mechanism, including surface scattering and volume scattering. These extreme cases need to be further investigated. Additionally, underestimation of AIEM with $m$-value of 0.05 at the incidence angles from 10 to 30 degrees for Ku-band and wind speed of $10 \mathrm{~m} /$ sand $15 \mathrm{~m} / \mathrm{s}$ (see Figure 8d,f) mentioned above indicates that the $m$-value may be not a constant but a function of incidence angles and should be smaller than 0.05 at these incidence angles, which will be notable and need further improvement as well.

\section{Conclusions}

In this paper, we quantitatively evaluate the roughness scales that are responsible, or contribute to, for radar backscattering from multi-scale sea surface. Such a physical process is called the frequency selective effect. Various scale sizes of roughness are extracted from different portions of spectral component in wave spectrum models and then casted into AIEM model to simulate backscattering coefficients at L, C, and Ku-bands for different incidence angles, wind speeds and wind directions. Simulation results are compared with corresponding radar measurements to determine the size of roughness (effective roughness) contributing to radar backscattering.

It is found that the effective roughness is selective from a portion of sea spectrum components $\left[k_{L}, \infty\right)$, with the lower limit $k_{L}$ proportional to the effective wavenumber $K$ determined by radar frequency and observation configuration $\left(K=2 k \sin \theta_{i}\right)$. The scale coefficient is 0.05 and independent on frequency, incident angle, wind speed, wind direction, and sea spectrum model. In addition, the effective roughness becomes rougher as the wind speed increases at medium and large incident angles (e.g., greater than 30 degrees) and the correlation length of effective roughness is about 3.2 times proportional to the effective wavelength $\lambda_{e}\left(\lambda_{e}=\lambda / 2 \sin \theta_{i}\right)$ regardless of incident angles and wind speeds for all three exploring frequencies. The simulated backscattering coefficients based on Apel spectrum at $\mathrm{Ku}$-band at high wind speeds (i.e., $10 \mathrm{~m} / \mathrm{s}$ and $15 \mathrm{~m} / \mathrm{s}$ ) and large incident angles (i.e., greater than 30 degrees) deviate from the radar measurements, which may be mainly attributed from the sea spectrum model itself.

Before concluding the paper, it is worth noting the effect of sea spectrum model on the scattering simulations (see Figures 8 and 9) and that a more comprehensive comparison between other sea spectra is necessary. Furthermore, the skewness effect caused by the non-Gaussian sea surface height has an influence on the upwind-downwind asymmetry of backscattering observed by radar at medium 
incidence angles (see Figure 5) but this is not considered in this paper, which will be investigated further in the future.

Author Contributions: K.-S.C. and D.X. conceived and developed this paper; D.X. performed the numerical simulations and wrote the paper; K.-S.C. and J.Z. modified and polished this paper.

Funding: This research was funded in part by National Key Research and Development Program of China under Grant 2018YFA0605403, and in part by the National Natural Science Foundation of China under grant 41531175. The work of Jiangyuan Zeng was supported by the Youth Innovation Promotion Association CAS under Grant 2018082.

Acknowledgments: The authors would like to thank the Royal Netherlands Meteorological Institute (KNMI) scatterometer team for providing the codes of CMOD7 and NSCAT-4 GMFs, which are available online: http: // projects.knmi.nl/scatterometer/.

Conflicts of Interest: The authors declare no conflict of interest.

\section{References}

1. Donelan, M.A.; Dobson, F.W.; Smith, S.D.; Anderson, R.J. On the dependence of sea-surface roughness on wave development. J. Phys. Oceanogr. 1993, 23, 2143-2149. [CrossRef]

2. Garrison, J.L.; Komjathy, A.; Zavorotny, V.U.; Katzberg, S.J. Wind speed measurement using forward scattered GPS signals. IEEE Trans. Geosci. Remote Sens. 2002, 40, 50-65. [CrossRef]

3. Bourlier, C. Azimuthal harmonic coefficients of the microwave backscattering from a non-Gaussian ocean surface with the first-order SSA model. IEEE Trans. Geosci. Remote Sens. 2004, 42, 2600-2611. [CrossRef]

4. Meissner, T.; Wentz, F.J.; Ricciardulli, L. The emission and scattering of L-band microwave radiation from rough ocean surfaces and wind speed measurements from the Aquarius sensor. J. Geophys. Res. Oceans 2014, 119, 6499-6522. [CrossRef]

5. Fore, A.; Yueh, S.H.; Tang, W.; Stiles, B.W.; Hayashi, A.K. Combined active/passive retrievals of ocean vector wind and sea surface salinity with SMAP. IEEE Trans. Geosci. Remote Sens. 2016, 54, 7396-7404. [CrossRef]

6. Alsweiss, S.O.; Jelenak, Z.; Chang, P.S. Remote Sensing of Sea Surface Temperature Using AMSR-2 Measurements. IEEE. Sel. Top. Appl. Earth Observ. Remote Sens. 2017, 10, 3948-3954. [CrossRef]

7. Ulaby, F.T.; Long, D.G. Microwave Radar and Radiometric Remote Sensing; University Michigan Press: Ann Arbor, MI, USA, 2014.

8. Tsang, L.; Kong, J.A.; Shin, R.T. Scattering of Electromagnetic Waves: Numerical Simulations; Wiley: Hoboken, NJ, USA, 2000; Volume 2.

9. Fung, A.K. Backscattering from Multiscale Rough Surfaces with Application to Wind Scatterometry; Artech House: Norwood, MA, USA, 2015.

10. Manninen, A.T. Multiscale surface roughness and backscattering Summary. J. Electromagn. Waves Appl. 1997, 11, 471-475. [CrossRef]

11. Mattia, F.; Toan, T.L. Backscattering properties of multiscale rough surfaces. J. Electromagn. Waves Appl. 1999, 13, 493-527. [CrossRef]

12. Fung, A.K.; Kuo, N.C. Backscattering from multiscale and exponentially correlated surfaces. J. Electromagn. Waves Appl. 2006, 20, 3-11. [CrossRef]

13. Wentz, F.J. A two scale scattering model with application to the JONSWAP '75 aircraft microwave scatterometer experiment. NASA Contract Rep. 1977, 2919, 122.

14. Jackson, F.C.; Walton, W.T.; Hines, D.E.; Walter, B.A.; Peng, C.Y. Sea surface mean square slope from Ku-band backscatter data. J. Geophys. Res. 1992, 97, 11411-11427. [CrossRef]

15. Plant, W.J. A two-scale model of short wind-generated waves and scatterometry. J. Geophys. Res. Oceans 1986, 91, 10735-10749. [CrossRef]

16. Donelan, M.A.; Pierson, W.J. Radar scattering and equilibrium ranges in wind-generated waves with application to scatterometry. J. Geophys. Res. 1987, 92, 4971-5029. [CrossRef]

17. Elfouhaily, T.; Chapron, B.; Katsaros, K.; Vandemark, D. A unified directional spectrum for long and short wind-driven waves. J. Geophys. Res. 1997, 102, 15781-15796. [CrossRef]

18. Durden, S.; Vesecky, J. A physical radar cross-section model for a wind-driven sea with swell. IEEE J. Ocean. Eng. 1985, 10, 445-451. [CrossRef] 
19. Apel, J.R. An improved model of the ocean surface wave vector spectrum and its effects on radar backscatter. J. Geophys. Res. Oceans 1994, 99, 16269-16291. [CrossRef]

20. Guerin, C.A.; Johnson, J.T. A simplified formulation for the crosspolarized backscattering coefficient under the second-order small slope approximation. IEEE Trans. Geosci. Remote Sens. 2015, 53, 6308-6314. [CrossRef]

21. Fois, F.; Hoogeboom, P.; Le Chevalier, F.; Stoffelen, A. An investigation on sea surface wave spectra and approximate scattering theories. In Proceedings of the International Geoscience and Remote Sensing Symposium, Quebec City, QC, Canada, 13-18 July 2014; pp. 4366-4369. [CrossRef]

22. Fung, A.K.; Chen, K.S. Microwave Scattering and Emission Models for Users; Artech House: Norwood, MA, USA, 2010.

23. Qiao, T.; Tsang, L.; Vandemark, D.; Yueh, S.H.; Liao, T.; Nouguier, H.F.; Chapron, B. Sea Surface Radar Scattering at L-Band Based on Numerical Solution of Maxwell's Equations in 3-D (NMM3D). IEEE Trans. Geosci. Remote Sens. 2018, 56, 3137-3147. [CrossRef]

24. Ulaby, F.T.; Moore, R.K.; Fung, A.K. Microwave Remote Sensing: Active and Passive; Artech House: Norwood, MA, USA, 1986; Volume 2.

25. Tsang, L.; Kong, J.A.; Shin, R.T. Theory of Microwave Remote Sensing; Wiley: Hoboken, NJ, USA, 1985.

26. Fung, A.K.; Pan, G.W. A scattering model for perfectly conducting random surfaces I. Model development. Int. J. Remote Sens. 1987, 8, 1579-1593. [CrossRef]

27. Durden, S.L.; Vesecky, J.F. A numerical study of the separation wavenumber in the two-scale scattering approximation (ocean surface radar backscatter). IEEE Trans. Geosci. Remote Sens. 1990, 28, 271-272. [CrossRef]

28. Awada, A.; Ayari, M.Y.; Khenchaf, A.; Coatanhay, A. Bistatic scattering from an anisotropic sea surface: Numerical comparison between the first-order SSA and the TSM models. Wave Random Media 2006, 16, 383-394. [CrossRef]

29. Chen, K.S.; Fung, A.K.; Weissman, D.E. A backscattering model for ocean surface. IEEE Trans. Geosci. Remote Sens. 1992, 30, 811-817. [CrossRef]

30. Fung, A.K. Microwave Scattering and Emission Models and Their Applications; Artech House: Norwood, MA, USA, 1994.

31. Wu, T.D.; Chen, K.-S. A reappraisal of the validity of the IEM model for backscattering from rough surfaces. IEEE Trans. Geosci. Remote Sens. 2004, 42, 743-753. [CrossRef]

32. Wu, T.D.; Chen, K.S.; Shi, J.; Lee, H.-W.; Fung, A.K. A study of an AIEM model for bistatic scattering from randomly rough surfaces. IEEE Trans. Geosci. Remote Sens. 2008, 46, 2584-2598. [CrossRef]

33. Chen, K.L.; Chen, K.S.; Li, Z.L.; Liu, Y. Extension and validation of an advanced integral equation model for bistatic scattering from rough surfaces. Prog. Electromagn. Res. 2015, 152, 59-76. [CrossRef]

34. Brogioni, M.; Pettinato, S.; Macelloni, G.; Paloscia, S.; Pampaloni, P.; Pierdicca, N.; Ticconi, F. Sensitivity of bistatic scattering to soil moisture and surface roughness of bare soils. Int. J. Remote Sens. 2010, 31, 4227-4255. [CrossRef]

35. Chen, K.S.; Wu, T.D.; Tsang, L.; Li, Q.; Shi, J.; Fung, A.K. Emission of rough surfaces calculated by the integral equation method with comparison to three-dimensional moment method simulations. IEEE Trans. Geosci. Remote Sens. 2003, 41, 90-101. [CrossRef]

36. Yueh, S.H.; Dinardo, S.; Fore, A.; Li, F.K. L-band passive and active microwave geophysical model functions of ocean surface winds and applications to Aquarius retrieval. IEEE Trans. Geosci. Remote Sens. 2013, 51, 4619-4632. [CrossRef]

37. Du, Y.; Yang, X.; Chen, K.S.; Ma, W.; Li, Z. An Improved Spectrum Model for Sea Surface Radar Backscattering at L-Band. Remote Sens. 2017, 9, 776. [CrossRef]

38. Stoffelen, A.; Verspeek, J.A.; Vogelzang, J.; Verhoef, A. The CMOD7 geophysical model function for ASCAT and ERS wind retrievals. IEEE J. Sel. Top. Appl. Earth Obs. Remote Sens. 2017, 10, 2123-2134. [CrossRef]

39. Royal Netherlands Meteorological Institute. NSCAT-4 Geophysical Model Function. Available online: http:/ / projects.knmi.nl/scatterometer/nscat_gmf/ (accessed on 1 March 2016).

40. Fung, A.K.; Zuffada, C.; Hsieh, C.Y. Incoherent bistatic scattering from the sea surface at L-band. IEEE Trans. Geosci. Remote Sens. 2001, 39, 1006-1012. [CrossRef]

41. Caulliez, G.; Guérin, C.A. Higher-order statistical analysis of short wind wave fields. J. Geophys. Res. Oceans 2012, 117, C06002-1-14. [CrossRef] 
42. Stogryn, A. Equations for calculating the dielectric constant of saline water (correspondence). IEEE Trans. Microw. Theory Tech. 1971, 19, 733-736. [CrossRef]

43. Cox, C.; Munk, W. Statistics of the sea surface derived from sun glitter. J. Mar. Res. 1954, 13, 198-227.

44. Voronovich, A.G. Small-slope approximation for electromagnetic wave scattering at a rough interface of two dielectric half-spaces. Waves Random Media 1994, 4, 337-367. [CrossRef]

45. Voronovich, A.G.; Zavorotny, V.U. Theoretical model for scattering of radar signals in Ku- and C-bands from a rough sea surface with breaking waves. Waves Random Media 2001, 11, 247-269. [CrossRef]

(C) 2019 by the authors. Licensee MDPI, Basel, Switzerland. This article is an open access article distributed under the terms and conditions of the Creative Commons Attribution (CC BY) license (http:/ / creativecommons.org/licenses/by/4.0/). 